\title{
Extracting psychiatric stressors for suicide from social media using deep learning
}

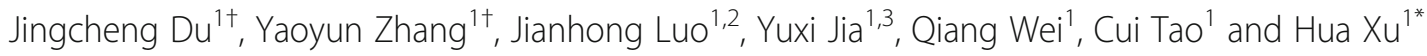

From The 2nd International Workshop on Semantics-Powered Data Analytics

Kansas City, MO, USA. 13 November 2017

\begin{abstract}
Background: Suicide has been one of the leading causes of deaths in the United States. One major cause of suicide is psychiatric stressors. The detection of psychiatric stressors in an at risk population will facilitate the early prevention of suicidal behaviors and suicide. In recent years, the widespread popularity and real-time information sharing flow of social media allow potential early intervention in a large-scale population. However, few automated approaches have been proposed to extract psychiatric stressors from Twitter. The goal of this study was to investigate techniques for recognizing suicide related psychiatric stressors from Twitter using deep learning based methods and transfer learning strategy which leverages an existing annotation dataset from clinical text.

Methods: First, a dataset of suicide-related tweets was collected from Twitter streaming data with a multiple-step pipeline including keyword-based retrieving, filtering and further refining using an automated binary classifier. Specifically, a convolutional neural networks (CNN) based algorithm was used to build the binary classifier. Next, psychiatric stressors were annotated in the suicide-related tweets. The stressor recognition problem is conceptualized as a typical named entity recognition (NER) task and tackled using recurrent neural networks (RNN) based methods. Moreover, to reduce the annotation cost and improve the performance, transfer learning strategy was adopted by leveraging existing annotation from clinical text.

Results \& conclusions: To our best knowledge, this is the first effort to extract psychiatric stressors from Twitter data using deep learning based approaches. Comparison to traditional machine learning algorithms shows the superiority of deep learning based approaches. CNN is leading the performance at identifying suicide-related tweets with a precision of $78 \%$ and an F-1 measure of 83\%, outperforming Support Vector Machine (SVM), Extra Trees (ET), etc. RNN based psychiatric stressors recognition obtains the best F-1 measure of 53.25\% by exact match and 67. 94\% by inexact match, outperforming Conditional Random Fields (CRF). Moreover, transfer learning from clinical notes for the Twitter corpus outperforms the training with Twitter corpus only with an F-1 measure of 54.9\% by exact match. The results indicate the advantages of deep learning based methods for the automated stressors recognition from social media.
\end{abstract}

Keywords: Suicide, Mental health, Psychiatric stressors, Social media, Deep learning, Named entity recognition

\footnotetext{
* Correspondence: hua.xu@uth.tmc.edu

${ }^{\dagger}$ Jingcheng Du and Yaoyun Zhang contributed equally to this work.

${ }^{1}$ The University of Texas School of Biomedical Informatics, 7000 Fannin St

Suite 600, Houston, TX 77030, USA

Full list of author information is available at the end of the article
}

(c) The Author(s). 2018 Open Access This article is distributed under the terms of the Creative Commons Attribution 4.0 International License (http://creativecommons.org/licenses/by/4.0/), which permits unrestricted use, distribution, and reproduction in any medium, provided you give appropriate credit to the original author(s) and the source, provide a link to the Creative Commons license, and indicate if changes were made. The Creative Commons Public Domain Dedication waiver (http://creativecommons.org/publicdomain/zero/1.0/) applies to the data made available in this article, unless otherwise stated. 


\section{Background}

Suicide has been one of the leading causes of deaths in the United States [1]. An average of 44,965 Americans die by suicides each year [2]. The national cost of suicides and suicidal behavior in the United States was $\$ 93.5$ billion in 2013 after adjustment for under-reporting [3]. According to the National Institute of Mental Health, the total suicide rate has increased $24 \%$ over the past 15 years [4]. Suicide and suicidal behaviors not only cause unbearable impacts on the specific individuals and surviving family and friends, but also create long lasting effects on whole communities [5].

One of the first steps toward suicide prevention is the identification of risk factors and causes associated with suicide [6].The multiple causes of suicide and suicidal behaviors can be broadly divided into stressors or triggers and predisposition [7]. As one of the major causes to suicide, psychiatric stressors are psychosocial or environmental factors that can profoundly impact cognition, emotion, and behavior of people [8]. The causes of suicide and suicidal behaviors can be complex and vary greatly from individual to individual. The identification of psychiatric stressors is critical to understanding the causes of potential suicide and suicidal behaviors for a specific individual, which is critical to provide a tailored and precise intervention strategy. For example, if the primary stressor for an individual is identified as school bullying, we can offer intensive individual interventions that provide the victim with individual support through meetings with students and parents, counseling, and sustained child and family support [9].

Mentions of stressors are often embedded in narratives such as clinical text or social media posts, and thus need to be recognized first for further investigation. Fortunately, the advances in machine learning and natural language processing (NLP) provide great opportunities to access mental health issues from large-scale narrative data. For example, Zhang et al. mined Electronic Health Records (EHR) to extract psychiatric stressors and symptoms from clinical text $[8,10]$. In recent years, social media has shown significant value for many public health related issues [11], as well as the influence on mental health and suicide-related behaviors [12, 13]. The wide popularity of social media provides unprecedented opportunities to access mental health and suicide risk from a large-scale population. The real-time information sharing flow on social media allows potential early detection and intervention for at-risk users.

Most previous studies focused on analyzing the association between suicidal ideation and the linguistic features of the contents (i.e. lexical analysis [14-18]) or posting behaviors (i.e. posting frequency $[5,19]$ ) on social media platforms. Some recent efforts attempted to classify tweets by levels of distress [6], concerns [20, 21] or types of suicidal communication [22] using machine learning or rule based approaches. However, few studies have been done mining the risk factors from social media. Jashinsky et al. tracked suicide risk factors from Twitter using keywords-based approaches [5]. We explored the association between psychiatric stressors and symptoms in tweets based on domain terminologies using Elasticsearch in the paper published in BIBM SEPDA 2017 workshop [23]. However, the keywords-based approaches often retrieve many irrelevant tweets, which introduce much noise into the further analyses.

As an extension to the conference paper, we narrowed down the focus of this paper to automatic identification of suicide related psychiatric stressors and propose a complete deep learning based pipeline to extract psychiatric stressors for suicide from Twitter data. This pipeline first collected and filtered suicide related tweets using keywords. Then, a convolutional neural network (CNN) based classifier removed more noise by further filtering out irrelevant tweets. After that, we applied a recurrent neural network (RNN) based algorithm to extract the mentions of stressors from texts of the suicide related tweets. Furthermore, given that it is time-consuming and costly to build an annotation dataset of stressors in tweets, we also examined the impact of transfer learning based approaches by leveraging pre-trained neural network layers training on clinical notes. To the best of our knowledge, it is the first effort to use systematic machine learning based approaches to extract psychiatric stressor from social media. It could have significant impacts on how people identify those in need of mental health services such as suicide prevention.

\section{Methods}

\section{System overview}

As illustrated in Fig. 1, the pipeline of the psychiatric stressor recognition from Twitter consisted of four steps. First, we retrieved a coarse set of suicide-related tweets using suicide-related keywords. Here we define the suicide related tweets as the tweets that contain the potential suicidal ideations, suicide history or plan etc. for the Twitter users. Second, we generated a refined candidate set of suicide related tweets by filtering tweets with the obvious stop words collected manually. Third, considering that keywords based tweets collection will introduce much noise, we employed a deep learning based classification model to further select out the precise suicide related tweets. Specifically, the CNN based approach was employed in this step. Finally, we detected the mentions of psychiatric stressors from the suicide-related tweets generated from the previous step. This problem was conceptualized as a named entity recognition (NER) task and we leveraged a state-of-art RNN based framework for this step. In addition, we further investigated transfer 


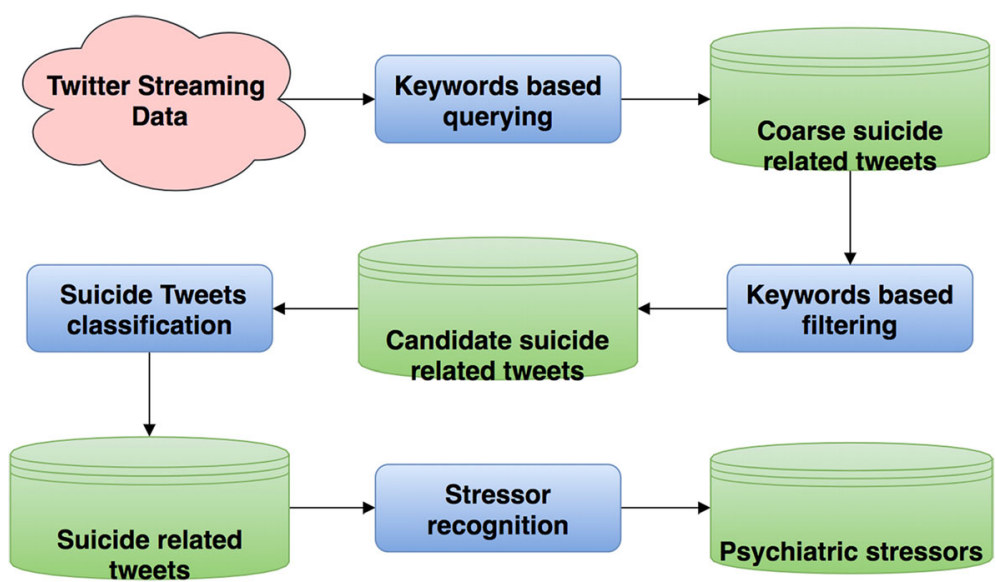

Fig. 1 Pipeline of suicides related psychiatric stressor recognition from Twitter

learning strategy to improve the performance of stressor extraction.

\section{Tweets collection and filtering}

We manually curated a suicide related keywords/phrases list to collect public suicide related tweets from June 26th, 2017 to Oct. 19th, 2017 through Twitter streaming API. The keywords/phrases list contains 21 keywords/phrases, such as "suicide", "kill myself", "want death", etc. While manually reviewing the collected tweets, we found most of the tweets were discussing news or advertisements instead of personal ideation or experience. It would have created higher annotation burden and would have deteriorated the performance of the machine learning system if we used the collected tweets directly. To create a refined candidate set of more precise suicide related tweets, we removed all the tweets that contained an URL or keywords such as "hotline", "suicide bomb", "suicide attack", etc. during the collection period. We inductively generated the stop words list to filter the tweets. By doing so, we observed a strong increase in the relevant tweets, which is beneficial for the training and evaluation of the deep learning system. The full list of keywords and stop keywords can be seen in Table 1. After filtering, 1,962,766 tweets were collected during this time period.

\section{Tweets annotation}

To identify tweets related to suicide and recognize the psychiatric stressors from tweets, two types of information were annotated. The first type of information is the label of the tweets. We annotated true suicide related tweets by choosing the label from Positive/Negative. Positive means the tweet is related to suicide or suicide ideation of the Twitter user (personal experience or feeling); tweets with the Negative label can be further categorized as 1) not related to suicide or suicide ideation, 2) the negation of suicide or suicide ideation (e.g. I don't want suicide), 3) the discussion of suicide or suicide ideation of other people, 4) the news or reports, 5) other non-positive tweets. Sample tweets with positive and negative labels are illustrated in Table 2.

The second type of information is the mention of psychiatric stressors in tweets text. For the tweets annotated with label Positive (i.e., suicide-related), we further annotated the mentions of psychiatric stressors. Some examples of stressors are listed in Table 3.

Here we divided this annotation process into two rounds: we first assigned Positive/Negative labels to 3263 tweets. We then trained a binary deep learning based classifier. We will introduce the classifier in the next section. We used this classifier to further select 3000 additional suicide related tweets with Positive labels that were relatively more precise than the original set. In the second round of annotation, we annotated the Positive/ Negative labels as well as the mentions of psychiatric stressors on the new 3000 tweets. We leveraged the Clinical Language Annotation, Modeling, and Processing Toolkit (CLAMP) for the annotation process [24].

\section{CNN based binary classification to recognize suicide related tweets}

As one deep learning based algorithm commonly used in various computer vision tasks [25], CNN also demonstrated excellent performance in many NLP tasks, including various text classification tasks [26-29]. We leveraged a classic CNN model for short text classification proposed by Kim et al. [26] to build the tweets binary classifier. We cleaned the tweets using the script from Stanford [30]. Then, we converted the tokens in each tweet to one-hot vectors and mapped the one-hot vectors to pre-trained GloVe Twitter embedding. The mapped embedding was used as the initial input feature to the $\mathrm{CNN}$ model. For the $\mathrm{CNN}$ model training, various filters were applied to generate the convolutional layers. 
Table 1 Examples of suicide-related keywords as queries for tweets retrieval and stop keywords used for irrelevant tweets filtering

\begin{tabular}{ll}
\hline Keywords & Stop Keywords \\
\hline "suicide", "suicidal", "suic", "self-harm", "bomb", "suicide attack", "suicide \\
"self-injury", "self harm", "self injury", \\
"hang myself", "hung myself", "kill & "suickide hotline", "https://", \\
myself", "kills myself", "killed myself", "http://" & "take my life", "takes my life", "want \\
to die", "wanted to die", "wants to \\
die", "want death", "wants death", \\
"wanted death", "to be dead"
\end{tabular}

We applied the max pooling strategy on the feature maps generated by different filters. We added dropout on the pooling layer to avoid overfitting. The pooling layer was connected to a fully connected layer with softmax output. The architecture of the $\mathrm{CNN}$ framework is shown in Fig. 2.

\section{RNN-based named entity recognition for stressors}

We conceptualized the stressor recognition as a NER task and leveraged a state-of-art RNN framework proposed by Dernoncourt el al $[31,32]$ to extract the mentions of stressors. The architecture of the framework is shown in Fig. 3. The token embedding mapped each token in the tweets text to a token vector. The character embedding mapped the characters in each token to character vectors. The character Bi-LSTM took the character embedding at each time step as the input and outputs the summary of each character for each token. The token embedding was concatenated with the character LSTM output and then fed to the token Bi-LSTM layer together. On top of token Bi-LSTM, we applied a sequential CRF to jointly decode labels for the whole tweet.

Table 2 Examples of tweets labeled with Positive or Negative in terms of relatedness to suicide

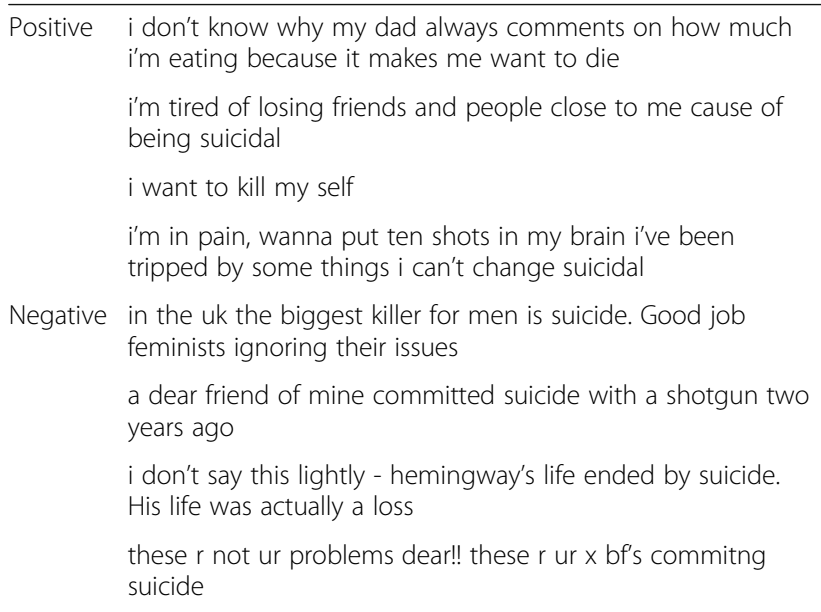

Transfer learning-based stressor recognition from tweets Transfer learning has proven to be an effective technique to improve the performance on a target task with limited annotation data, by using some knowledge learned from a source task [33, 34]. Instead of training the model for a target task from a completely blank network, transfer learning can re-use all or some of the parameters trained from a source task. In our previous studies on extraction of stressors from clinical notes [8, 10], we created a dataset with stressors annotations from the psychiatric notes provided by the CEGS N-GRID 2016 challenge organizers [35]. The annotated dataset contained 946 sentences with stressors annotation. For the transfer learning based approach, clinical notes were used as the source domain to transfer stressor related knowledge to the target domain: Twitter.

\section{Experiment configuration}

The core of the deep learning based framework in this study had two modules: a CNN based binary classifier to select suicide related tweets and a RNN based NER system to extract the mentions of stressors. The following experiments were performed for these two modules separately.

For the binary classifier, we trained the CNN model in two steps using two rounds of annotations, respectively. We first trained a CNN model on the first round of annotations with Positive/Negative labels. To improve the classifier performance on the unbalanced class distribution (623 Positives/3263 tweets), especially on the Positive class (suicide related), the training corpus was built with equal sample sizes of the Positive class and the Negative class (Positive: 498 tweets, Negative: 498 tweets); the evaluation corpus was built using the same distribution of origin tweets class (Positive: 125 tweets, Negative: 652 tweets). We further used the best classifier on the evaluation corpus to select another 3000 candidate suicide related tweets that potentially have a high proportion of Positive labels for the second round of annotation. The labeled tweets in the later 3000 candidate tweets were divided into training, validation and testing sets with a proportion of $7: 1: 2$.

Table 3 Examples of psychiatric stressors annotation from the suicide-related tweets. Bold refers to the annotated stressors

job hunting makes me want to commit suicide Imao

honestly every time $\mathrm{i}$ think about me getting pregnant $\mathrm{i}$ wanna kill myself

i just realized that i was completely sexually assaulted by some

disgusting photographer and i want to fucking kill myself

well i guess it's too bad that i'm just young adult without a degree about to lose my job and probably planning my suicide afterwards

school and work make me want to die everyday 


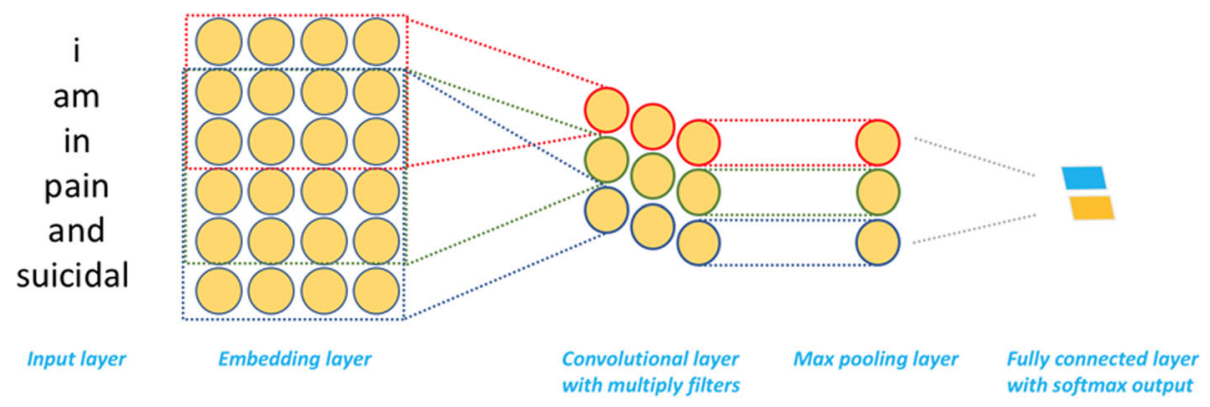

Fig. 2 The architecture of CNN based binary classifier for suicide related labels prediction

The CNN based classifier was trained and evaluated based on the three sets. We used the GloVe Twitter embedding to initialize the embedding layer of $\mathrm{CNN}$ and compare the performance using dimensions at 50, 100, and 200 respectively. In order to confirm the superiority of the CNN model, we also evaluated several traditional machine learning algorithms, including Extra Trees (ET), Random Forest (RF), Logistics Regression (LR) and Support Vector Machine (SVM) with Radial Basis Function (RBF) as the kernel, as well as a Bidirectional Long Short-Term Memory (Bi-LSTM) classifier. All the algorithms use GloVe Twitter embedding (dimension 50) as the input. The traditional classification algorithms take the average of the word vectors of the tweet text as the input feature. We evaluated the performance of the classifiers using standard metrics, including precision, recall and F-measure.

For the RNN based NER, we performed the following experiments:

1. To demonstrate the superiority of the RNN model, we used classic Conditional Random Fields (CRF) as the baseline model. We leveraged CLAMP for implementation [24]. Typical features for named entity recognition in CLAMP were employed including lexical features (e.g., bag-of-word, cases, prefix/suffix/stem patterns), syntactic features (e.g. Part of Speech tags), context features (e.g., ngrams), distributional representation of words (e.g., brown clustering, word embedding), and domain knowledge features (e.g., semantic types in UMLS), etc.

2. Performance comparison of different GloVe Twitter embedding dimensions at 50, 100, and 200 on stressor recognition tasks. Performance of different embedding dimensions were evaluated and reported using standard metrics, including precision, recall and F-measure, based on exact match (same entity boundary) and inexact match (overlap in entity boundary) respectively.

3. Performance comparison between using transfer learning strategy and training on Twitter data only for stressor recognition. For the transfer learning approach, clinical notes were used as the source domain to transfer stressor related knowledge to the target Twitter domain. The tweets with Positive label in the second round of annotation were divided into training, validation and testing set with

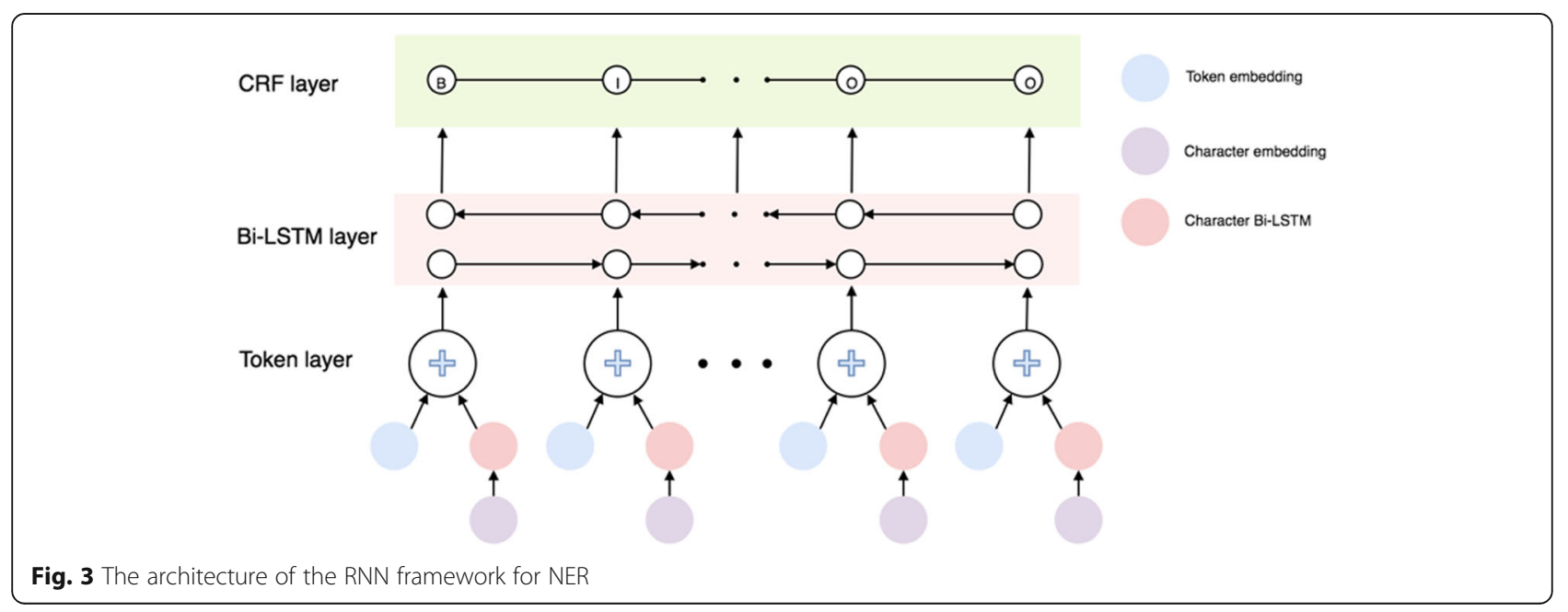


a proportion of 6: 2: 2. Specifically, the following experiments were conducted:

a. Performance comparison using different sizes of annotated tweets for training in the transfer learning strategy and training on Twitter data only. We evaluated the performance of using different training data sizes from $5,10,20,30$, 40,50 to $60 \%$ (all training data) respectively. The validation and testing datasets were kept with the same configuration consistently. We reported the standard metrics, including precision, recall and F-measure, based on exact match and inexact match respectively.

b. Performance comparison of transferring pretrained parameters up to different layers of the RNN framework, from token embedding layer, character embedding layer, character LSTM layer, token LSTM layer, to fully connected layer to the final CRF layer.

\section{Results}

\section{Annotation of suicide related tweets and psychiatric stressors}

In the first round of annotation, we assigned the Positive/Negative labels to 3263 tweets. Among these tweets, only 623 tweets were annotated as Positive (suicide related). We trained the binary classifier (P: 0.66, R: 0.79, F: 0.72 ) on this annotated dataset and further selected 3000 tweets for the second round of annotation. Among these 3000 tweets, 1985 tweets were annotated as Positive, and 2162 stressor entitles were annotated in the Positive tweets.

\section{Suicide-related tweet classification}

Table 4 lists the performance of suicide-related tweets classification, using the $\mathrm{CNN}$ based algorithm and GolVe Twitter embedding features of different dimensions. As shown, the Positive type had a high recall (0.90 as the optimal). It also achieved an overall F-measure sufficient ( 0.83 as the optimal) for practical applications.

Table 5 lists the comparison of the CNN based algorithm with traditional machine learning algorithms as well as the Bi-LSTM model, using GloVe Twitter embedding (dimension 50) as the input. As shown, the CNN model led the performance in Positive type, Negative type and the overall accuracy. Bi-LSTM was second to the $\mathrm{CNN}$ model.

\section{Psychiatric stressor extraction}

\section{Performance of RNN-based stressor recognition}

In our pilot work, we evaluated the impact of different types of word embedding for stressors recognition. We first set embedding dimension at 100 and compared GloVe Twitter embedding, GloVe embedding and
Table 4 Experimental performance of suicide-related tweets classification, using the CNN based algorithm and word embedding features of different dimensions. Bold number denotes the largest number in that row

\begin{tabular}{lllll}
\hline & & $\mathrm{D}=50$ & $\mathrm{D}=100$ & $\mathrm{D}=200$ \\
\hline Precision & Positive & 0.78 & 0.76 & $\mathbf{0 . 7 9}$ \\
\multirow{3}{*}{ Recall } & Negative & 0.69 & $\mathbf{0 . 7 0}$ & 0.65 \\
& Positive & 0.88 & $\mathbf{0 . 9 0}$ & 0.84 \\
F-1 measure & Negative & 0.51 & 0.45 & $\mathbf{0 . 5 6}$ \\
& Positive & $\mathbf{0 . 8 3}$ & 0.82 & 0.81 \\
& Negative & 0.59 & 0.55 & $\mathbf{0 . 6 0}$ \\
\hline
\end{tabular}

MIMIC embedding. GloVe Twitter embedding achieved best exact match and inexact match F-measure at 53.25 and $65.46 \%$ respectively. As a result, we chose GloVe Twitter embedding for further analysis. We further evaluated the impact of dimensions of the word embedding. The dimension at 100 achieves best exact match F-1 measure (53.25\%), while the dimension at 50 achieves best inexact match F-1 measure (67.94\%). Although the CRF baseline model led the precision of stressor recognition, however, as we can observe, the RNN based models greatly improved the recall, thus achieving better F-1 measure. The details of metric scores are in Table 6.

\section{Performance of transfer learning -based stressor recognition}

Initialized with GloVe Twitter embedding with dimensions of 100 , the best model trained on clinical notes achieved precision of $53.08 \%$, recall of $47.51 \%$ and F-1 measure of $50.14 \%$ on the clinical notes validation set. We transferred parameters up to different layers from this model to initialize the RNN model training on Twitter dataset.

We first investigated the impact of varying training set sizes of the Twitter dataset (see Fig. 4). For both learning strategies, F-measure improved as more training samples were used. Transfer learning improved the F-measure

Table 5 Performance comparison of the CNN model with other algorithms. SVM: Support Vector Machine; ET: Extra Trees; RF:

Random Forest; LR: Logistics Regression; Bi-LSTM: Bi-directional Long Short-Term Memory. Bold number denotes the largest number in that row

\begin{tabular}{llllllll}
\hline \multirow{2}{*}{ Precision } & & CNN & SVM & ET & RF & LR & Bi-LSTM \\
\cline { 3 - 4 } & $\mathbf{0 . 7 8}$ & 0.7 & 0.69 & 0.69 & 0.7 & 0.73 \\
Recall & Negative & 0.69 & $\mathbf{0 . 7 2}$ & 0.58 & 0.5 & 0.67 & 0.65 \\
& Positive & 0.88 & $\mathbf{0 . 9 6}$ & 0.94 & 0.88 & 0.94 & 0.9 \\
F-1 measure & Negative & $\mathbf{0 . 5 1}$ & 0.21 & 0.17 & 0.24 & 0.23 & 0.37 \\
& Positive & $\mathbf{0 . 8 3}$ & 0.81 & 0.79 & 0.77 & 0.8 & 0.81 \\
Accuracy & Negative & $\mathbf{0 . 5 9}$ & 0.33 & 0.27 & 0.33 & 0.34 & 0.47 \\
\hline
\end{tabular}


Table 6 Experimental performance metric of stressors recognition on different types of word embedding for both exact and inexact match. CRF: Conditional Random Fields. Bold number denotes the largest number in that column

\begin{tabular}{lllllll}
\hline & \multicolumn{2}{l}{ Precision } & Recall & & \multicolumn{2}{l}{ F-1 measure } \\
\hline & exact & inexact & exact & inexact & exact & inexact \\
GloVe Twitter 50 & 0.4868 & 0.6843 & 0.4765 & $\mathbf{0 . 6 7 4 5}$ & 0.4816 & $\mathbf{0 . 6 7 9 4}$ \\
Glove Twitter 100 & 0.5822 & 0.7123 & 0.4906 & 0.6057 & $\mathbf{0 . 5 3 2 5}$ & 0.6546 \\
GloVe Twitter 200 & 0.5248 & 0.6808 & $\mathbf{0 . 4 9 7 7}$ & 0.6484 & 0.5108 & 0.6642 \\
CRF & $\mathbf{0 . 6 0 0}$ & $\mathbf{0 . 7 8 4}$ & 0.398 & 0.572 & 0.478 & 0.661 \\
\hline
\end{tabular}

over training with Twitter data only, though the improvement diminished as more training samples were used. This phenomena is consistent with transfer learning on other NER tasks [34]. Compared to training with Twitter data only, transfer learning strategy can save on the number of annotations to achieve the same level of performance. For example, as shown in Fig. 4, transfer learning using $30 \%$ of the Twitter training data can achieved a higher F-measure than the baseline strategy where $40 \%$ of the training data was used.

Figures 5 and 6 show the impact of transferring the parameters up to each layer of the RNN model measured by exact match and inexact match respectively. For exact match, transferring the layers up to character LSTM achieved best F-measure at 54.9\% (see Fig. 7), compared with $53.25 \%$ achieved by the model without transfer learning. Transferring all layers could also increase the F-measure, but not as much as transferring some lower layers only. As for inexact match, transferring the layers up to the character LSTM layer achieved a F-measure very close to transferring all layers (67.47 to
$67.51 \%$ ), out-performing by $\sim 0.02$ the model without transfer learning (F-measure, 65.46\%).

\section{Discussion}

In this paper, we proposed and evaluated a systematic pipeline to extract psychiatric stressors for suicide and suicidal ideations from Twitter. This pipeline had multiple steps: 1) Curation of a precise suicide related Twitter corpus by using keywords to collect and filter, and a deep learning based classifier to further select out suicide related tweets. This deep learning based classifier achieved a good F-measure at $83 \%$, which is sufficient for practical application. 2) Leverage a state-of-art RNN based framework to extract stressors from suicide related tweets. This framework achieved the best F-measure by exact match at $53.25 \%$. We also investigated the impact of transfer learning from clinical notes for the Twitter corpus. We found that transfer learning can achieve the same level of performance while reducing the annotation cost of tweets, in comparison with using only the Twitter data for training. For this RNN based framework, we found that transferring the parameters up to the character LSTM layer achieved the best F-measure by exact match (54.9\%), 1.65\% higher than the best model without transfer learning (F-measure: $53.25 \%)$.

To our best knowledge, this is the first effort to extract psychiatric stressors from Twitter data using deep learning based approaches. Compared with lexicon based text analysis, which often introduces much noise, this framework will facilitate more precise analysis. The deep learning based framework also saves great effort on feature engineering, compared to the conventional machine

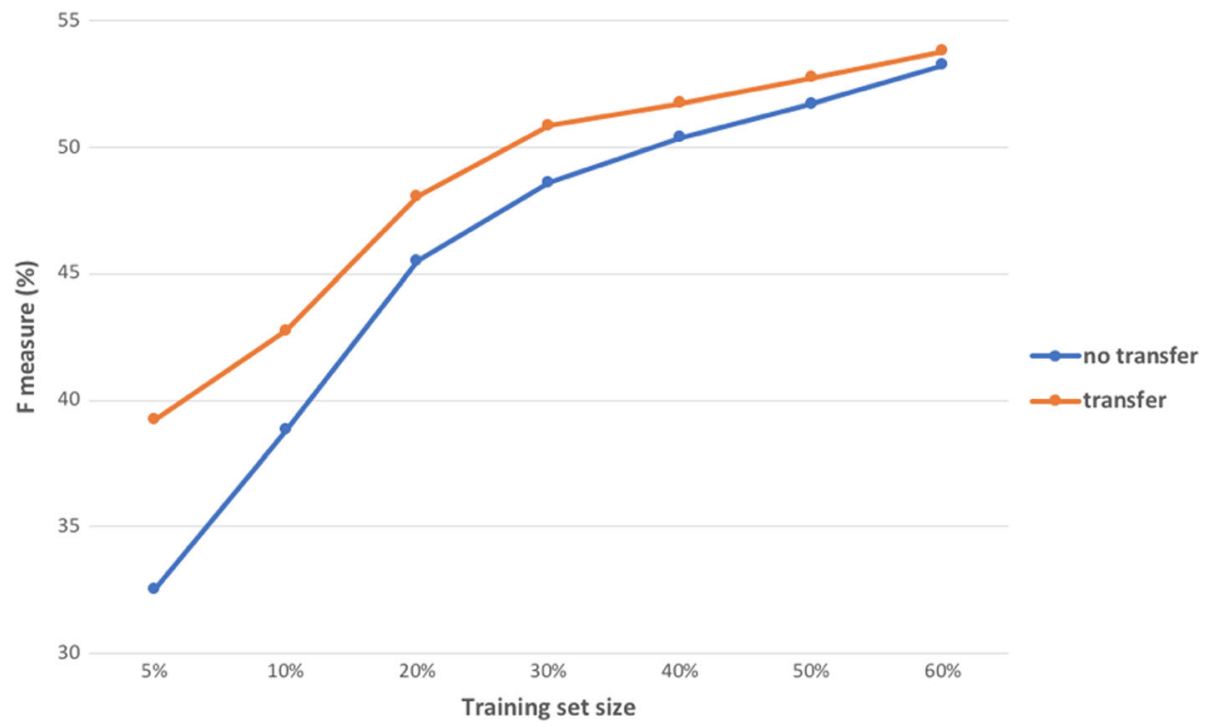

Fig. 4 Impact of transfer learning on the size of training data measured on F-1 measure by exact match 


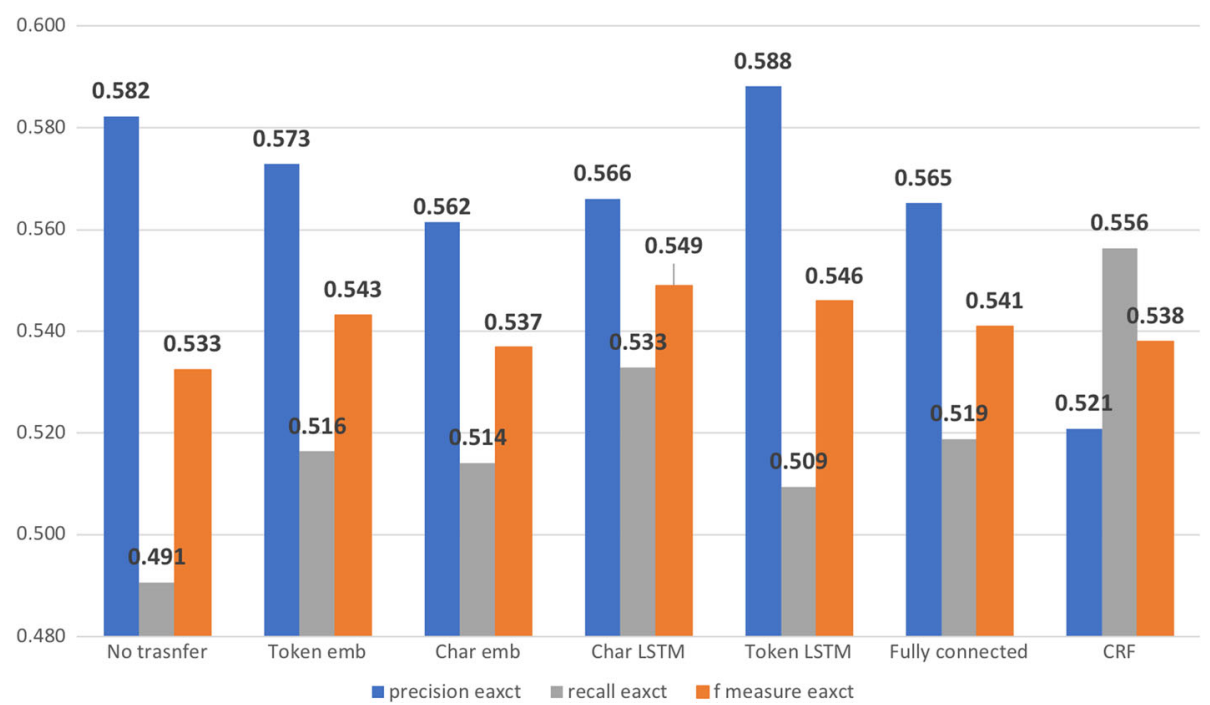

Fig. 5 Impact of transferring the parameters up to each layer of the RNN model for stressors recognition measured by exact match

learning based approaches that require many hand-crafted features. A common limitation of machine learning based Twitter data analysis is the highly imbalanced dataset [28, 36]. We mitigated the impact of imbalanced distributions of classes and entities by building a precise suicide related Twitter corpus using multiple steps. Using state-of-art deep learning based approaches and the transfer learning strategy, our pipeline $o$ achieved a reasonable performance on both suicide related tweets prediction as well as psychiatric stressors extraction.

Certain limitations remain for our study. The major limitation of this study is the lack of ground truth data for the exact mental health status of the Twitter users. The annotations were based on the contents of the tweets. As tweet contents may not always reflect the true mental health status, our dataset may include some false positive suicide related tweets. In addition, due to the limitation of data collection, the current analysis is only based on a single tweet. The lack of context of tweets may introduce inexact interpretations of the contents. There also still exists room for performance improvement on stressor recognition task. The most common type of prediction error in stressor recognition is the boundary issue. As illustrated in Table 7, causes of prediction errors also include (1) missing annotations in the gold standard, (2) the lack of negation detection and (3) mistakenly predicting high-frequent mentions of stressors within a wrong context.

This study obtained a modest performance (exact match F-measure: 54.9\%) on the stressors recognition

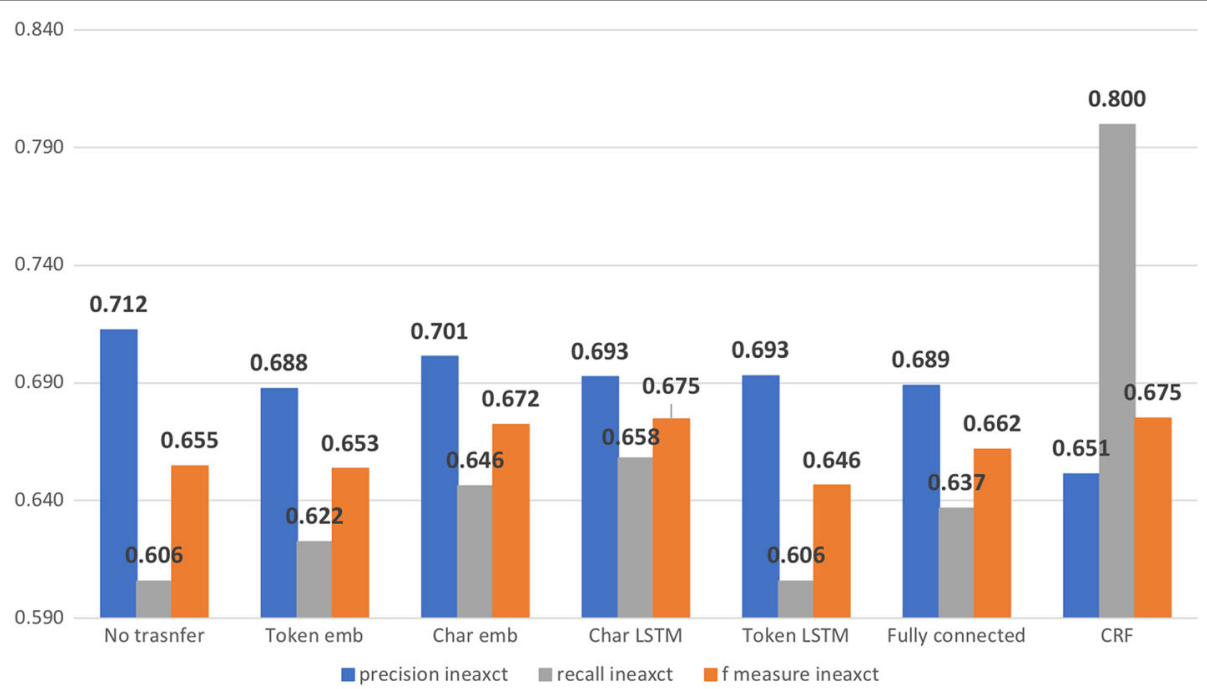

Fig. 6 Impact of transferring the parameters up to each layer of the RNN model for stressors recognition measured by inexact match 


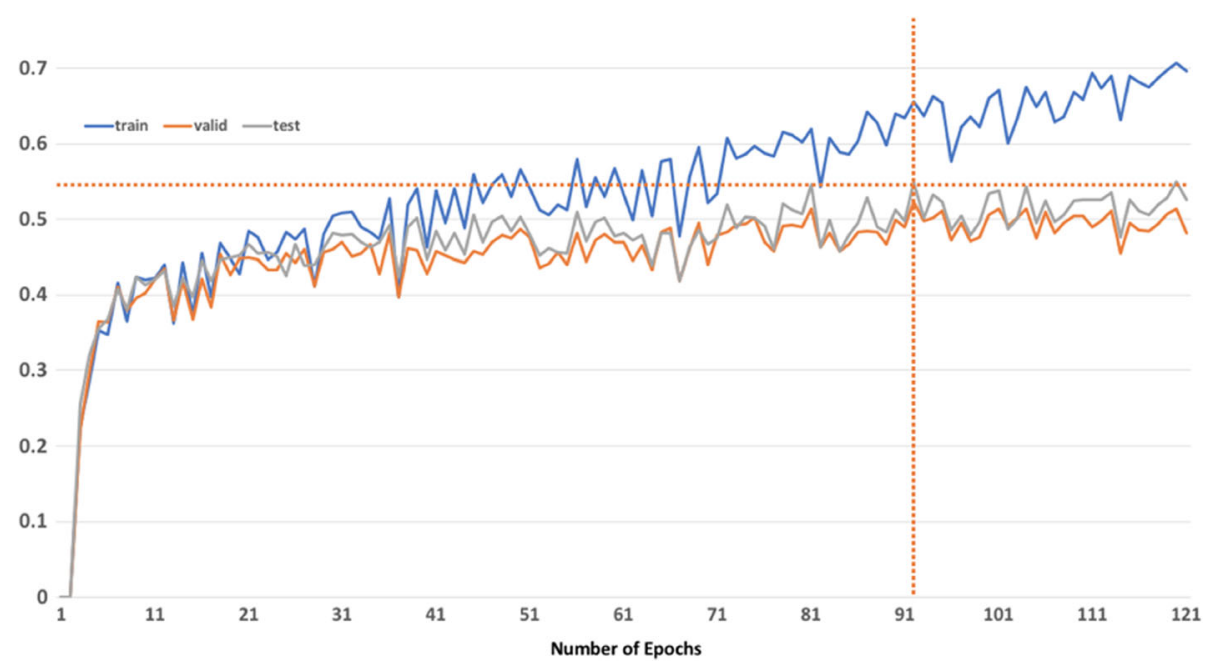

Fig. 7 Exact match F-1 measure by each epoch from the model transferring up to character LSTM layer

task using deep learning based methods. In fact, Twitter NER tasks appear to be more challenging than NER tasks in other domains. For example, in the recent two Twitter NER challenges [37, 38], most of the state-of-art systems achieved F-1 measure between 40 to $60 \%$ on various entity recognition tasks, including person, location, etc. Considering stressors recognition is an even more complicated problem (i.e. various lengths of boundary, sparseness of expression patterns), we posit that our result is comparable to the state-of-art performances achieved so far.

As for the future work, we're in the process of preparing a large-scale suicide related Twitter dataset. We will access the user level information by analyzing the historical tweets instead of the single tweet without any context. Ground truth data showing the mental health status of the users will be acquired by crowdsourcing or linkage to the users' electrical health record (EHR) data. To improve the performance of the stressors recognition, we will further refine the quality of the gold standard annotation. Moreover, the deep learning based NER systems will be augmented with domain knowledge based and context based rules for further performance improvement.

\section{Conclusions}

Few automated approaches have been proposed to extract psychiatric stressors from Twitter, mainly due to (a) the lack of annotated corpora that are time-consuming and costly to build, and (b) the inherent linguistic difficulties that stressors present beyond well-defined clinical concepts such as diseases. To our best knowledge, this is the first and comprehensive effort to extract psychiatric stressors from Twitter data using deep learning based approaches. We've build an annotated Twitter corpus on suicide related stressor recognition. We also performed extensive experiments to justify the use of the approaches presented. The comparison to traditional machine learning algorithms showed the superiority of deep learning based approaches. Our methods demonstrated good performance at identifying suicide-related tweets with a F-1 measure of $83 \%$. In addition, stressor recognition obtained the best F- 1 measure of $53.25 \%$ by exact match and $67.94 \%$ by inexact match. Moreover, transfer learning strategy was

Table 7 Common types of prediction errors. Bold: annotated entity; Underline: predicted entity

\begin{tabular}{|c|c|}
\hline Boundary & $\begin{array}{l}\text { - when the bottom of my foot inches i want death. } \\
\text { - allen high school makes me want to kill myself for not allowing me to get out of a 2nd math class (that i don't } \\
\text { need) knowing i work } \\
\text { - hormonal birth control made me suicidal and acne-ridden! there's no winning against the beast within me }\end{array}$ \\
\hline $\begin{array}{l}\text { Annotation error: missing } \\
\text { annotation }\end{array}$ & - harassment is not a thing you should have fun with. it almost killed me yesterday. \\
\hline Negation & - im confident in my maths but phys makes me want to die i want \\
\hline Entity in wrong context & - gonna kill myself after work today. Cant take the bullying no longer. \\
\hline False negative & $\begin{array}{l}\text { - when will my best friend stop telling me every little things she does with her boyfriend. i want death } \\
\text { - i'd kill myself to make everybody pay }\end{array}$ \\
\hline
\end{tabular}


found to further improve the performance and show potential for saving annotation effort. The results indicate the potential to use deep learning based methods for automated stressor recognition in social media.

\section{Abbreviations}

Bi-LSTM: Bidirectional long short-term memory; CLAMP: Clinical language annotation, modeling, and processing toolkit; CNN: Convolutional neural networks; CRF: Conditional random fields; ET: Extra trees; LR: Logistics regression; LSTM: Long short-term memory; NER: Named entity recognition; NLP: Natural language processing; RBF: Radial basis function; RF: Random forest; RNN: Recurrent neural networks; SVM: Support vector machines

\section{Acknowledgments}

We thank the anonymous reviewers for their careful reading of our manuscript and their many insightful comments.

\section{Funding}

Research was partially supported by the National Library of Medicine of the National Institutes of Health under Award Number 2R01LM010681-05 and R01 LM011829, the National Institute of Allergy and Infectious Diseases of the National Institutes of Health under Award Number R01 Al130460, the National Cancer Institute of the National Institutes of Health under Award Number U01 CA180940, and the Cancer Prevention Research Institute of Texas (CPRIT) Training Grant \#RP160015. The authors also acknowledge the support from the National Nature Science Foundation of China (Grant No.71501172), Zhejiang Provincial Nature Science Foundation of China (Grant No.LY18G020017), and the scholarship from the China Scholarship Council. Publication of this article was supported by National Institutes of Health under Award Number 2R01LM010681-05.

\section{Availability of data and materials}

The datasets generated during and/or analyzed during the current study are not publicly available due to time issues but are available from the corresponding author on reasonable request.

\section{Disclaimer}

The content is solely the responsibility of the authors and does not necessarily represent the official views of the National Library of Medicine and the Cancer Prevention and Research Institute of Texas.

\section{About this supplement}

This article has been published as part of BMC Medical Informatics and Decision Making Volume 18 Supplement 2, 2018: Selected extended articles from the 2nd International Workshop on Semantics-Powered Data Analytics. The full contents of the supplement are available online at https://bmcmedinformdecismak.biomedcentral.com/articles/supplements/volume-18-supplement-2.

\section{Authors' contributions}

$J D$ and $Y Z$ designed the study, collected the data, performed the experiments and drafted the manuscript. JD, YZ, JL, YJ and QW designed the annotation quideline. JD, YZ, JL and $Y J$ annotated the data. $C T$ and $H X$ supervised the study. Everyone read and revised the manuscript. All authors read and approved the final manuscript.

\section{Ethics approval and consent to participate}

This study received IRB exemption from Committee for the Protection of Human Subjects at The University of Texas Health Science Center at Houston. The reference number is HSC-SBMI-17-0900.

\section{Competing interests}

Dr. Hua Xu is a founder of Melax Technologies Inc., which licenses the CLAMP software.

\section{Publisher's Note}

Springer Nature remains neutral with regard to jurisdictional claims in published maps and institutional affiliations.

\section{Author details}

${ }^{1}$ The University of Texas School of Biomedical Informatics, 7000 Fannin St Suite 600, Houston, TX 77030, USA. ${ }^{2}$ Department of Management Science and Engineering, Zhejiang Sci-Tech University, Hangzhou 310018, China.

${ }^{3}$ Department of Medical Informatics, School of Public Health, Jilin University, Changchun 130021, Jilin, China.

Published: 23 July 2018

\section{References}

1. Wasserman D. Suicide: an unnecessary death. Oxford: Oxford University Press; 2016.

2. American Foundation for Suicide Prevetion. Suicide Statistics [Internet]. Available from: https://afsp.org/about-suicide/suicide-statistics/. Accessed Feb 2018.

3. Shepard DS, Gurewich D, Lwin AK, Reed GA, Silverman MM. Suicide and suicidal attempts in the United States: costs and policy implications. Suicide life-threatening Behav Wiley Online Library. 2016;46:352-62.

4. National Insitute of Mental Health. Suicide [Internet]. Available from: https:// www.nimh.nih.gov/health/statistics/suicide/index.shtml. Accesses Feb 2018.

5. Jashinsky J, Burton SH, Hanson CL, West J, Giraud-Carrier C, Barnes MD, et al. Tracking suicide risk factors through twitter in the US. Crisis Hogrefe Publishing. 2014;35:51.

6. Homan CM, Johar R, Liu T, Lytle M, Silenzio V, Alm CO. Toward macroinsights for suicide prevention: analyzing fine-grained distress at scale. Acl. 2014;2014:107.

7. Mann JJ, Apter A, Bertolote J, Beautrais A, Currier D, Haas A, et al. Suicide prevention strategies: a systematic review. Jama. American Medical Association. 2005;294:2064-74.

8. Zhang $\mathrm{OR}$, Zhang $Y, X U$ J, Roberts $K$, Zhang $X Y, X u ~ H$. Interweaving Domain Knowledge and unsupervised learning for psychiatric stressor extraction from clinical notes. Int. Conf. Ind. Eng. Other Appl. Appl. Intell. Syst 2017. p. 396-406.

9. Feinberg T. Bullying prevention and intervention. Princ Leadersh Mag. 2003;4:10-4.

10. Zhang $Y$, Zhang $\mathrm{O}, \mathrm{Wu} Y$, Lee $\mathrm{H}-\mathrm{J}, \mathrm{Xu} J$, Xu H, et al. Psychiatric symptom recognition without labeled data using distributional representations of phrases and on-line knowledge. J. Biomed. Inform. Elsevier. 2017;

11. Moorhead SA, Hazlett DE, Harrison L, Carroll JK, Irwin A, Hoving C. A new dimension of health care: systematic review of the uses, benefits, and limitations of social media for health communication, J Med Internet Res, vol. 15. Toronto, Canada: JMIR Publications Inc; 2013. p. 1-16.

12. Luxton DD, June JD, Fairall JM. Social media and suicide: a public health perspective. Am J Public Health American Public Health Association. 2012; 102:S195-200.

13. Wongkoblap A, Vadillo MA, Curcin V. Researching mental health disorders in the era of social media: systematic review. J Med Internet Res JMIR Publications Inc. 2017;19

14. Lv M, Li A, Liu T, Zhu T. Creating a Chinese suicide dictionary for identifying suicide risk on social media. PeerJ PeerJ Inc. 2015;e1455:3.

15. Coppersmith G. Wood a. Exploratory analysis of social media prior to a suicide attempt. In: Proc. 3rd work. Comput. Linguist. Clin. Psychol. From linguist. Signal to Clin. Real; 2016. p. 106-17.

16. Huang X, Zhang L, Chiu D, Liu T, Li X, Zhu T. Detecting suicidal ideation in Chinese microblogs with psychological lexicons. In: Proc - 2014 IEEE Int. Conf. Ubiquitous Intell. Comput. 2014 IEEE Int. Conf. Auton. Trust. Comput. 2014 IEEE Int. Conf. Scalable Comput. Commun. Assoc. Sy; 2014. p. 844-9.

17. Cheng Q, Li TMH, Kwok C-L, Zhu T, Yip PSF. Assessing suicide risk and emotional distress in Chinese social media: A text mining and machine learning study. J Med Internet Res JMIR Publications Inc. 2017;19:e243.

18. Zhang L, Huang X, Liu T, Chen Z, Zhu T, Li A, et al. Using linguistic features to estimate suicide probability of Chinese microblog users. Int Conf Hum Centered Comput 2014;1-11.

19. Smith RJ, Crutchley P, Schwartz HA, Ungar L, Shofer F, Padrez KA, et al. Variations in facebook posting patterns across validated patient health conditions:a prospective cohort study. J Med Internet Res. 2017;19:1-11.

20. O'Dea B, Wan S, Batterham PJ, Calear AL, Paris C, Christensen H. Detecting suicidality on twitter. Internet Interv Elsevier. 2015;2:183-8.

21. Abboute A, Boudjeriou Y, Entringer G, Azé J, Bringay S, Poncelet P. Mining twitter for suicide prevention. In: Int Conf Appl Nat Lang to Data Bases/ Information Syst; 2014. p. 250-3. 
22. Burnap P, Colombo G, Amery R, Hodorog A, Scourfield J. Multi-class machine classification of suicide-related communication on twitter. Online Soc. Netw Media. Elsevier. 2017;2:32-44.

23. Du J, Zhang Y, Tao C, Xu H. A pilot study of mining association between psychiatric stressors and symptoms in tweets. IEEE Int Conf Bioinforma Biomed. 2017:2017:1254-7.

24. Soysal E, Wang J, Jiang M, Wu Y, Pakhomov S, Liu H, et al. CLAMP-a toolkit for efficiently building customized clinical natural language processing pipelines. J. Am. Med. In: Informatics Assoc; 2017.

25. LeCun Y, Kavukcuoglu K, Farabet C. Convolutional networks and applications in vision. Circuits Syst. In: (ISCAS), Proc. 2010 IEEE Int Symp, vol. 2010. p. 253-6.

26. Kim Y. Convolutional neural networks for sentence classification. arXiv Prepr. arXiv1408.5882. 2014;

27. Dos Santos CN, Gatti M. Deep convolutional neural networks for sentiment analysis of short texts. In: COLING; 2014. p. 69-78.

28. Nakov P, Ritter A, Rosenthal S, Sebastiani F, Stoyanov V. SemEval-2016 task 4: sentiment analysis in twitter. In: Proc SemEval; 2016. p. 1-18.

29. Conneau A, Schwenk H, Le Cun Y, Barrault L Very Deep Convolutional Networks for Text Classification arXiv Prepr arXiv160601781. 2016;

30. Paulus R. Script for preprocessing tweets [Internet]. Available from: http://nlp.stanford.edu/projects/glove/preprocess-twitter.rb. Accessed Feb 2018

31. Dernoncourt F, Lee JY, Szolovits P NeuroNER: an easy-to-use program for named-entity recognition based on neural networks arXiv Prepr arXiv1705.05487. 2017

32. Dernoncourt F, Lee JY, Uzuner O, Szolovits P. De-identification of patient notes with recurrent neural networks. J Am Med Informatics Assoc The Oxford University Press. 2016:ocw156.

33. Kermany DS, Goldbaum M, Cai W, Lewis MA, Valentim CCS, Liang H, et al. Identifying Medical Diagnoses and Treatable Diseases by Image-Based Deep Learning. Cell Elsevier. 2018;172:1122-1131.e9.

34. Lee JY, Szolovits P, Dernoncourt F, Szolovits P Transfer Learning for NamedEntity Recognition with Neural Networks arXiv Prepr arXiv1705.06273. 2017;

35. Stubbs A, Filannino M, Uzuner Ö. De-identification of psychiatric intake records: overview of 2016 CEGS N-GRID shared tasks track 1. J. Biomed. Inform. Elsevier. 2017:75:S4-S18

36. Du J, Xu J, Song H, Liu X, Tao C. Optimization on machine learning based approaches for sentiment analysis on HPV vaccines related tweets. J Biomed Semantics. 2017;8:9.

37. Derczynski L, Nichols E, van Erp M, Limsopatham N. Results of the WNUT2017 shared task on novel and emerging entity recognition. In: Proc. 3rd work. Noisy user-generated Text; 2017. p. 140-7.

38. Lopez C, Partalas I, Balikas G, Derbas N, Martin A, Reutenauer C, et al. CAp 2017 challenge: Twitter Named Entity Recognition. arXiv Prepr. arXiv1707. 07568. 2017.

\section{Ready to submit your research? Choose BMC and benefit from:}

- fast, convenient online submission

- thorough peer review by experienced researchers in your field

- rapid publication on acceptance

- support for research data, including large and complex data types

- gold Open Access which fosters wider collaboration and increased citations - maximum visibility for your research: over $100 \mathrm{M}$ website views per year 